\title{
Inferencia de indicadores de idoneidad didáctica a partir de orientaciones curriculares
}

\section{Evaluation of didactic suitability indicators from curricular orientations}

\author{
Juan D. Godino* \\ Hernán Rivas ${ }^{* *}$ \\ Pedro Arteaga ${ }^{* * *}$
}

\begin{abstract}
Resumen: La evaluación de la idoneidad didáctica de procesos de enseñanza y aprendizaje de las matemáticas requiere disponer de instrumentos adecuados que orienten de manera fundamentada dicha evaluación. En este trabajo describimos una metodología para la mejora progresiva de instrumentos de evaluación de la idoneidad de procesos de instrucción matemática mediante el análisis de contenido de propuestas curriculares. Las unidades de análisis son clasificadas según las facetas y componentes propuestos en la Teoría de la Idoneidad Didáctica para identificar normas e indicadores de idoneidad, los cuales son confrontados con el sistema propuesto por dicha teoría, a fin de identificar concordancias y complementariedades. Esta metodología es ejemplificada mediante su aplicación a los Principios y Estándares 2000 del NCTM en el área de contenido de estadística de los niveles K-8.
\end{abstract}

Palabras claves: Educación Matemática. Formación de profesores. Estándares de calidad.

\begin{abstract}
Assessment of didactic suitability of mathematics teaching and learning processes requires suitable instruments to guide and support it. This paper presents a methodology for progressive improvement of such instruments to evaluate the suitability of mathematical instruction processes based on the analysis of curricular orientations. Analysis units are classified according to the facets and components proposed by the Didactic Suitability Theory to identify norms and suitability indicators, which are compared with the system proposed by this theory, in order to point out agreements and complementarities. This methodology is exemplified through its application to the principles and standards of the National Council of Teachers of Mathematics (NCTM) in the statistics content area at the level K-8.
\end{abstract}

Keywords: Mathematical Instruction. Teachers' Education. Quality standards.

\footnotetext{
*Universidad de Granada. E-mail: <jgodino@ugr.es>

** Pontificia Universidad Católica de Chile, Sede Regional de Villarica. E-mail: <hrivasa@uc.cl>

*** Universidad de Granada. E-mail: <parteaga@ugr.es>
} 
Resumo: A avaliação da idoneidade didática de processos de ensino e aprendizagem das matemáticas requer dispor de instrumentos adequados que orientem tal avaliação de maneira fundamentada. Nesse artigo, descrevemos una metodologia para a melhoria progressiva de instrumentos de avaliação da idoneidade de processos de instrução matemática mediante a análise do conteúdo de propostas curriculares. As unidades de análise são classificadas segundo as facetas e componentes propostos na Teoria da Idoneidade Didática para identificar normas e indicadores de idoneidade, os quais são confrontados com o sistema proposto por essa teoria, a fim de identificar concordâncias e complementariedades. Essa metodologia é exemplificada mediante sua aplicação aos os princípios e padrões do National Council of Teachers of Mathematics (NCTM) na área do conteúdo de estatística dos níveis K-8.

Palavras-chave: Educação Matemática. Formação de professores. Padrões de qualidade.

\section{Problema y antecedentes}

El problema de qué enseñar y cómo enseñar ha sido y continúa siendo un problema central de la educación, en general, y por tanto también, de la Educación Matemática, cuando esta disciplina se concibe como una "ciencia de diseño" (WITTMAN, 1995; HJALMARSON; LESH, 2008; LESH; SRIRAMN, 2010). Este problema afecta a los responsables de los diseños curriculares, a los profesores de matemáticas y también a los formadores de profesores (qué matemáticas y didáctica enseñar a los futuros profesores, y cómo hacerlo). Aunque estas decisiones se deberían tomar de una manera fundamentada en los resultados de las investigaciones, de hecho se toman con frecuencia con el mejor juicio posible de las personas implicadas.

Consideramos que la noción de idoneidad didáctica introducida en el marco del Enfoque Ontosemiótico (EOS) del conocimiento y la instrucción matemática (GODINO; BATANERO; FONT, 2007; GODINO et al., 2007; GODINO et al., 2009) puede ser útil en el diseño, implementación y evaluación de procesos de enseñanza y aprendizaje de las matemáticas, así como en el diseño y evaluación de programas y acciones formativas de profesores.

Como se afirma en Godino et al. (2009, p. 60), los criterios de idoneidad se deben de entender como reglas de corrección emanadas del discurso argumentativo de la comunidad científica, cuando éste está orientado a conseguir un consenso sobre "lo que se puede considerar como mejor". Así mismo, reconocen que, "El sistema de componentes y descriptores de la idoneidad didáctica que hemos presentado no son una propuesta cerrada. Se trata de una herramienta cuya aplicación y discusión por los formadores de profesores, los propios profesores e investigadores permitirá su progresiva mejora y enriquecimiento" (GODINO et al., 2009, p. 60). 
En Godino (2011) se han sistematizado y ampliado los indicadores de idoneidad didáctica propuestos en trabajos previos (GODINO et al., 2007; GODINO et al., 2009), al tiempo que se relacionan dichos indicadores con principios didácticos asumidos por diversas teorías usadas en didáctica de las matemáticas, principalmente la Teoría de situaciones (BROUSSEAU, 1997) y la Matemática realista (FREUDENTHAL, 1991).

Queda abierta la necesidad de desarrollar "pautas de análisis y valoración de la idoneidad didáctica" para las distintas áreas de contenido matemático, entendidas como instrumentos que propongan de manera fundamentada indicadores de idoneidad de procesos de aprendizaje y enseñanza de las matemáticas. Es necesario hacer adaptaciones de instrumentos ya existentes para su aplicación en áreas de contenidos particulares, y además "fundamentar" los indicadores de manera explícita, bien en los resultados de investigaciones o bien en juicios expertos asumidos por la comunidad académica, frecuentemente plasmados en las directrices curriculares nacionales e internacionales.

Dado que el sistema de indicadores de idoneidad didáctica se puede entender y usar como un instrumento aplicable a la evaluación de procesos de instrucción matemática ${ }^{1}$ consideramos necesario ampliar y fundamentar dichos indicadores para asegurar su validez y fiabilidad como instrumento de medición. Teniendo en cuenta la diversidad de facetas y componentes que se deben considerar, y el carácter situado de la noción de idoneidad, parece necesario abordar el problema de la construcción, no de un único instrumento sino una familia de instrumentos orientados cada uno a un nivel educativo y contenidos matemáticos específicos. Esto no significa descartar que tales instrumentos compartan un núcleo común de principios didácticos generales en cada una de las facetas.

Nuestro objetivo en este trabajo es describir una estrategia metodológica para la extracción o inferencia de indicadores de idoneidad didáctica a partir de las directrices curriculares, poniéndola en práctica en el análisis de los Principios y Estándares del NCTM (2000). Los indicadores inferidos son contrastados y complementados con los indicadores propuestos en Godino (2011).

$\mathrm{El}$ análisis que realizamos de los Principios y Estándares (en adelante nos referimos a este documento como PE-2000) lo centramos en el reconocimiento de normas sobre la enseñanza y aprendizaje de las matemáticas en general (cualquier contenido y procesos), así como en aquellas que se refieren al contenido de análisis de datos y probabilidad. Esto permite elaborar dos sistemas de indicadores de idoneidad didáctica, para la matemática escolar en general y para el área específica de estadística.

\footnotetext{
${ }^{1}$ Entendemos la instrucción como proceso organizado de enseñanza y aprendizaje de contenidos específicos, no como un simple adiestramiento.
} 
Por limitaciones de espacio describimos con detalle la inferencia de indicadores de idoneidad epistémica (significados institucionales) y remitimos a Rivas (2011) donde se describe el proceso y los resultados para las facetas cognitiva, afectiva, interaccional, mediacional y ecológica, así como para las interacciones entre dichas facetas.

\section{Marco teórico y metodología}

El marco teórico que usamos en este trabajo es el Enfoque Ontosemiótico del conocimiento y la instrucción matemática (EOS) (GODINO, 2002; GODINO; BATANERO; FONT, 2007), y en particular la noción de idoneidad didáctica, la cual describimos sucintamente a continuación.

\section{La noción de idoneidad didáctica}

Como se afirma en Godino (2011) la noción de idoneidad didáctica, sus dimensiones, criterios y desglose operativo, han sido introducidos en el EOS como herramientas que permite el paso de una didáctica descriptiva explicativa a una didáctica normativa, esto es, una didáctica que se orienta hacia la intervención efectiva en el aula. Se considera que esta noción puede servir de punto de partida para una teoría de diseño instruccional (REIGELUTH, 2000) que tenga en cuenta, de manera sistémica, las dimensiones o facetas epistémica - ecológica, cognitiva - afectiva, interaccional - mediacional implicadas en los procesos de estudio de las áreas curriculares específicas.

La idoneidad didáctica de un proceso de instrucción se define como la articulación coherente y sistémica de las seis componentes siguientes, relacionada cada una de ellas con la faceta correspondiente de igual nombre (GODINO; BATANERO; FONT, 2007):

- Idoneidad epistémica, se refiere al grado de representatividad de los significados institucionales implementados (o pretendidos), respecto de un significado de referencia.

- Idoneidad ecológica, grado en que el proceso de estudio se ajusta al proyecto educativo del centro, la escuela y la sociedad y a los condicionamientos del entorno en que se desarrolla.

- Idoneidad cognitiva, expresa el grado en que los significados pretendidos/ implementados estén en la zona de desarrollo potencial de los alumnos, así como la proximidad de los significados personales logrados a los significados pretendidos/ implementados.

- Idoneidad afectiva, grado de implicación (interés, motivación,...) del alumnado en el proceso de estudio. La idoneidad afectiva está relacionada tanto con factores que dependen de la institución como con factores que dependen básicamente del alumno y de su historia escolar previa. 
- Idoneidad interaccional. Un proceso de enseñanza-aprendizaje tendrá mayor idoneidad desde el punto de vista interaccional si las configuraciones y trayectorias didácticas permiten, por una parte, identificar conflictos semióticos potenciales, y por otra parte permitan resolver los conflictos que se producen durante el proceso de instrucción.

- Idoneidad mediacional, grado de disponibilidad y adecuación de los recursos materiales y temporales necesarios para el desarrollo del proceso de enseñanza- aprendizaje.

Como indicadores de idoneidad epistémica Godino (2011) propone los incluidos en lo cuadro 1, clasificados según las entidades primarias que caracterizan el modelo epistémico-cognitivo del EOS: situaciones-problemas; elementos lingüísticos representacionales, elementos regulativos (conceptos/ definiciones, procedimientos y proposiciones); argumentos. También se resaltan las relaciones entre dichos elementos primarios que caracterizan la actividad matemática como esencialmente relacional.

Cuadro 1 - Componentes e indicadores de idoneidad epistémica

\begin{tabular}{|l|l|}
\hline COMPONENTES & \multicolumn{1}{|c|}{ INDICADORES $^{2}$} \\
\hline Situaciones-problemas & $\begin{array}{l}\text { a) Se presenta una muestra representativa y articulada de situaciones } \\
\text { de contextualización, ejercitación y aplicación } \\
\text { b) Se proponen situaciones de generación de problemas } \\
\text { (problematización) }\end{array}$ \\
\hline Lenguajes & $\begin{array}{l}\text { a) Uso de diferentes modos de expresión matemática (verbal, } \\
\text { gráfica, simbólica...), traducciones y conversiones entre los mismas. } \\
\text { b) Nivel del lenguaje adecuado a los niños a que se dirige } \\
\text { c) Se proponen situaciones de expresión matemática e interpretación }\end{array}$ \\
\hline $\begin{array}{l}\text { Reglas (Definiciones, } \\
\text { proposiciones, } \\
\text { procedimientos) }\end{array}$ & $\begin{array}{l}\text { a) Las definiciones y procedimientos son claros y correctos, y están } \\
\text { adaptados al nivel educativo al que se dirigen } \\
\text { b) Se presentan los enunciados y procedimientos fundamentales } \\
\text { del tema para el nivel educativo dado } \\
\text { c) Se proponen situaciones donde los alumnos tengan que generar } \\
\text { o negociar definiciones proposiciones o procedimientos }\end{array}$ \\
\hline Argumentos & $\begin{array}{l}\text { a) Las explicaciones, comprobaciones y demostraciones son } \\
\text { adecuadas al nivel educativo a que se dirigen } \\
\text { b) Se promueven situaciones donde el alumno tenga que argumentar }\end{array}$ \\
\hline Relaciones & $\begin{array}{l}\text { a) Los objetos matemáticos (problemas, definiciones, proposiciones, } \\
\text { etc.) se relacionan y conectan entre sí. }\end{array}$ \\
\hline
\end{tabular}

Fuente: Elaboración propia.

\footnotetext{
${ }^{2}$ Para facilitar la comparación de los indicadores de idoneidad propuestos en la Pauta-EOS, y los inferidos a partir de los PE-2000 usaremos las letras a), b), c) ..., para referir a los indicadores de la Pauta-EOS, y a'), b'), c') para los referidos a la Pauta-NCTM. La finalidad es elaborar una "pauta refinada" mediante la fusión de las pautas anterior, cuyos indicadores serán denotados con a"), b"), ...
} 
Por limitaciones de espacio en este trabajo centraremos la atención en la dimensión epistémica de la idoneidad didáctica. También incluiremos indicadores de idoneidad relacionados con la faceta ecológica (idoneidad ecológica) al estar estrechamente relacionada con la epistémica, e indicadores de las relaciones entre la faceta epistémica y las restantes facetas. Remitimos al lector al trabajo citado de Godino (2011) donde se incluyen indicadores de idoneidad para las diferentes facetas y otras interacciones ${ }^{3}$.

\section{Análisis de contenido}

En este estudio empleamos la técnica de análisis de contenido (cualitativo), para extraer y sistematizar las normas de idoneidad didáctica contenidas en una de las orientaciones curriculares más difundidas a nivel internacional. Se trata de los Principios y Estándares para la Educación Matemática del NCTM (2000). Dichas normas serán interpretadas como indicadores de idoneidad para las diversas facetas o dimensiones de los procesos de enseñanza y aprendizaje de las matemáticas.

En una primera fase el texto es dividido en unidades de análisis, las cuales son clasificadas según las facetas y componentes que propone la Teoría de la Idoneidad Didáctica. Seguidamente se procede a una segunda fase en la que dichas unidades son comparadas entre sí y reducidas con el fin de evitar reiteraciones. Posteriormente, del análisis de las normas extraídas de los PE-2000 se infieren indicadores de idoneidad didáctica de procesos de instrucción matemática, y particularizados en determinados casos para la estadística. Estos indicadores son comparados con los indicadores propuestos en Godino (2011), lo que da lugar a una versión revisada, y en su caso ampliada de dichos indicadores. La finalidad de la metodología descrita es la elaboración de una pauta o guía de indicadores de idoneidad "refinados", al tiempo que se identifican limitaciones y complementariedades entre ambos instrumentos.

Este proceso se puede repetir con nuevas orientaciones curriculares, así como con otras fuentes que aporten criterios de idoneidad didáctica; por ejemplo, dicha metodología ha sido seguida en Rivas (2011) al inferir indicadores de idoneidad didáctica a partir del análisis del currículo español de Educación Primaria (MEC, 2006).

\footnotetext{
${ }^{3}$ Nos referiremos a este sistema de indicadores como Pauta-EOS para distinguirla y compararla con el sistema de indicadores de idoneidad inferidos de los PE-2000, que designamos como Pauta-NCTM.
} 


\section{Normas epistémicas en los principios y estándares 2000}

En esta sección procedemos a inferir las normas, así como las explicaciones y justificaciones de las mismas, contenidas en los PE-2000 que en el marco del EOS son consideradas como epistémicas, esto es, referidas a características de los conocimientos matemáticos contemplados desde el punto de vista institucional. Para ello hemos clasificado las unidades de contenido según las categorías: situaciones-problemas, elementos lingüísticos/representaciones, elementos regulativos (conceptos/definición, procedimientos, proposiciones), argumentos y relaciones ${ }^{4}$.

A lo largo de esta sección se observará que las normas, justificaciones o explicaciones inferidas del análisis de los PE-2000, en ocasiones harán referencia a contenidos generales de las matemáticas, válidos también para el caso de la estadística, y en otras ocasiones serán exclusivas de contenidos de estadística y probabilidad.

\section{Situaciones-problema}

En las unidades de análisis que hacen referencia al papel de las situaciones-problemas en el diseño de los planes de instrucción matemática y al logro del aprendizaje, hemos observado que algunas de ellas expresan con claridad una posición normativa sobre dicho uso, otras tratan de explicar en qué consiste la orientación hacia los problemas, mientras que otras unidades aportan razones por las cuales se requiere el uso de la resolución de problemas. En este caso las unidades de análisis o enunciados son generales para cualquier área de las matemáticas y en particular para la estadística.

Seguidamente hacemos una primera reducción de los datos suprimiendo redundancias y clasificando los enunciados según su carácter de norma, explicación o justificación ${ }^{5}$.

Normas:

- (Se deben) "Aplicar y adaptar una variedad de estrategias para resolver problemas" (NCTM, 2003, p. 120).

- (Se debe) "Construir nuevos conocimientos matemáticos a través de la resolución de problemas" (NCTM, 2003, p. 120).

\footnotetext{
${ }^{4}$ Debido a la cantidad de unidades de análisis en las distintas componentes de esta faceta no las incluiremos todas en el artículo; hacemos una selección evitando repeticiones y unidades que expresan ideas similares. Remitimos al lector a Rivas (2011) para disponer de dicha información.

${ }^{5}$ Las páginas de cada unidad corresponden al texto de la traducción al español del NCTM (2000) realizada por la Sociedad Andaluza de Educación Matemática Thales.
} 
- "La resolución de problemas no es un tema aparte, sino un proceso que debería impregnar el estudio de las matemáticas y proporcionar un contexto en el que se aprendan los conceptos y destrezas" (NCTM, 2003, p. 186).

Las normas anteriores, como otras que contienen ideas similares, pueden ser sintetizadas en las siguientes normas de carácter general, que en el caso de este componente epistémico viene expresada en el estándar de proceso sobre la "resolución de problemas":

Los programas de enseñanza de todas las etapas deberían capacitar a todos los estudiantes para:

- construir nuevos conocimientos a través de la resolución de problemas;

- resolver problemas que surjan de las matemáticas y de otros contextos;

- aplicar y adaptar diversas estrategias para resolver problemas;

- controlar el proceso de resolución de los problemas matemáticos y reflexionar sobre él ${ }^{6}$.

Explicaciones:

- "La resolución de problemas es una característica notable de la actividad matemática y un medio importante para desarrollar el conocimiento matemático" (NCTM, 2003, p. 120).

- "La esencia de la resolución de problemas es saber qué hacer al enfrentarse con problemas no familiares" (NCTM, 2003, p. 264).

Justificaciones:

- "La resolución de problemas da oportunidades para usar y ampliar el conocimiento de los conceptos de todos los Estándares de contenidos" (NCTM, 2003, p. 120).

- "Los buenos problemas pueden inspirar la exploración de ideas matemáticas importantes" (NCTM, 2003, p. 186).

- "La resolución de problemas es fundamental para la investigación y la aplicación de las matemáticas” (NCTM, 2003, p. 260).

\section{Elementos lingüísticos/representaciones}

Llama la atención la insistencia de los PE-2000 en el uso de representaciones para representar conceptos, expresar relaciones matemáticas y resolver problemas.

\footnotetext{
${ }^{6}$ Esta norma incluye un aspecto metacognitivo, por lo que en el marco del EOS correspondería a la faceta mixta epistémica-cognitiva.
} 
A continuación incluimos las unidades de análisis referidas a este componente epistémico clasificándolas en los apartados de normas, explicaciones y justificaciones; hemos suprimido algunas repeticiones y refundido algunas de ellas. Primero incluimos las normas sobre representaciones de carácter general (para cualquier contenido matemático) y a continuación las que se refieren al contenido de estadística.

Normas:

Generales:

- "Es importante que los alumnos tengan oportunidades no sólo de aprender las formas convencionales de representación, sino también de construir, perfeccionar y usar sus propias representaciones" (NCTM, 2003, p. 72).

- "Deberían ampliarse los repertorios de representaciones para incluir dibujos más complejos, tablas, gráficas y palabras para modelizar problemas y situaciones" (NCTM, 2003, p. 73).

- (Se deben) "representar datos mediante objetos concretos, dibujos y gráficos" (NCTM, 2003, p. 112).

- "Seleccionar, aplicar y traducir representaciones matemáticas para resolver problemas" (NCTM, 2003, p. 210).

Las normas anteriores como también otras que contienen ideas similares pueden ser sintetizadas en las siguientes normas de carácter general:

- Se debe usar un amplio repertorio de representaciones (materiales, icónicas y simbólicas) para modelizar problemas e ideas matemáticas, realizando procesos de traducción entre las mismas.

- Se debe favorecer que los alumnos construyan, perfeccionen y usen sus propias representaciones.

Sobre estadística:

- "Los títulos y etiquetas utilizados en sus representaciones deberían identificar de forma clara qué datos se representan" (NCTM, 2003, p. 113).

- "reconocer las diferencias en la representación de datos cualitativos y cuantitativos" (NCTM, 2003, p. 180).

- "Deberían familiarizarse con diversas representaciones de datos; entre otras, las tablas, los diagramas de puntos, los diagramas de barras y los lineales" (NCTM, 2003, p. 182). 
- "seleccionar, crear y utilizar representaciones gráficas apropiadas de datos, incluyendo histogramas, gráficos de caja y nubes de puntos" (NCTM, 2003, p. 252).

- "utilizar frecuencias absolutas y relativas, diagramas de barras e histogramas para representar los datos que hayan reunido, y a decidir qué tipo de representación es la apropiada según el propósito" (NCTM, 2003, p. 254).

Estas normas y otras similares que han sido suprimidas en el proceso comparación y reducción se pueden sintetizar de la manera siguiente:

El programa de estudio debe incluir situaciones en las que,

- Se usen diversas representaciones de datos, tales como, tablas, diagramas de puntos, diagramas de barras y lineales, gráfico de sectores y de cajas, nubes de puntos. Estos gráficos deben incluir los títulos y etiquetas que permitan identificar claramente los datos representados.

- Sea necesario discriminar las situaciones en las cuales es más adecuada una u otra representación.

Explicaciones y justificaciones:

Encontramos en los PE-2000 unidades de análisis que explican las normas anteriores y otras que aportan justificaciones de las mismas. Incluimos a continuación ejemplos de tales unidades de análisis, distinguiendo también explicaciones y justificaciones de las normas sobre representaciones para cualquier contenido matemático (generales), o bien explicaciones y justificaciones específicas para el bloque de estadística.

Generales:

- "Usar el lenguaje de las matemáticas para expresar ideas matemáticas con precisión” (NCTM, 2003, p. 132).

- "Crear y utilizar representaciones para organizar, registrar y comunicar ideas matemáticas” (NCTM, 2003, p. 140).

- "El comprender y utilizar conceptos y procedimientos matemáticos se enriquece cuando los alumnos pueden traducir diferentes representaciones de una misma idea" (NCTM, 2003, p. 143).

Sobre estadística:

- "Comparar distintas representaciones ayuda a aprender a evaluar cómo éstas muestran aspectos importantes de los datos" (NCTM, 2003, p. 183). 


\section{Elementos regulativos}

Encontramos unidades de análisis en las que los PE-2000 proponen normas sobre el aprendizaje de conceptos matemáticos importantes, con profundidad y compresión, así como aplicar procedimientos, conceptos y procesos. También se concede un papel a la formulación de conjeturas (enunciados proposicionales), su refinamiento y comprobación.

\section{Normas sobre conceptos y procedimientos (matemáticos generales)}

En los estándares de contenido abundan las especificaciones sobre los tipos de conceptos y procedimientos que los estudiantes deben aprender. En la página 21 encontramos una indicación de que los estudiantes "deben ser capaces de aplicar los procedimientos, conceptos y procesos". En el estándar de proceso sobre resolución de problemas se menciona que se deben "aplicar y adaptar diversas estrategias para resolver problemas".

El detalle de los elementos regulativos que se deben incluir se indica para cada área de contenido. Así, para el caso de la estadística se resaltan:

Normas sobre conceptos y procedimientos estadísticos:

- "Los estudiantes deberían llegar a comprender los elementos básicos del análisis estadístico: seleccionar una muestra adecuada, recoger datos de esta muestra, describir la muestra y hacer inferencias razonables que relacionen la muestra y la población.” (NCTM, 2003, p. 53).

- "Empezando en la etapa 3-5 y continuado en los niveles medios, se debería pasar de analizar y describir un conjunto de datos a comparar dos o más conjuntos" (NCTM, 2003, p. 53).

- "Comprender y aplicar conceptos básicos de probabilidad" (NCTM, 2003, p. 112).

- "clasificar y ordenar utilizando, simultáneamente, más de un atributo" (NCTM, 2003, p. 114).

- "describir sucesos como probables o no probables, y discutir su grado de probabilidad usando expresiones como seguro, igualmente probable e improbable" (NCTM, 2003, p. 180).

- "predecir la probabilidad de resultados de experimentos sencillos, y someter a prueba tales predicciones" (NCTM, 2003, p. 180).

- "comprender la naturaleza de los dos tipos de datos: cualitativos $[\ldots]$ y cuantitativos" (NCTM, 2003, p. 182).

- "hallar, utilizar e interpretar medidas de centralización y de dispersión, incluyendo la media y el rango intercuartílico" (NCTM, 2003, p. 252). 
- "comparar la utilidad de la media y de la mediana como medidas de centralización para diferentes conjuntos de datos" (NCTM, 2003, p. 255).

Las normas señaladas y otras que evocan ideas similares pueden ser sintetizadas de la siguiente manera:

El programa de estudio debe incluir,

- Las nociones básicas del proceso de análisis estadístico elemental (seleccionar muestras adecuadas, recoger datos, describir y comparar muestras y hacer inferencias justificadas).

- Conceptos y procedimientos tales como: población, muestra, dato, variable, frecuencias, promedios (media, mediana y moda), medidas de dispersión (recorrido, desviación típica, rango intercuartílico), valor atípico.

- Conceptos y procedimientos básicos de probabilidad (grado de probabilidad de sucesos, asignación de probabilidades de resultados de experimentos sencillos).

Explicaciones y justificaciones de elementos regulativos (específicos de estadística):

- "Al principio, los niños trabajan más frecuentemente con datos censales; por ejemplo, con una encuesta sobre la clase de helados favorita de cada niño de la clase" (NCTM, 2003, p. 53).

- "A medida que los mayores empiezan a ver un conjunto de datos como un todo, necesitan herramientas para describirlo. Las medidas de centralización (media, mediana y moda) y de dispersión (rango, desviación típica), y los atributos sobre la forma de la distribución de datos llegan a ser útiles a los estudiantes como descriptores" (NCTM, 2003, p. 53).

- "construir un cierto conocimiento de la probabilidad y el azar haciendo experimentos con objetos concretos, tales como sacar fichas coloreadas de una bolsa" (NCTM, 2003, p. 54).

- "proponer preguntas y recoger datos relativos a ellos y a su entorno" (NCTM, 2003, p. 112).

- "distinguir el significado de los diferentes números: aquéllos que representan valores ("en mi familia hay cuatro personas"), de los que indican cuántas veces (frecuencia) se presenta un valor en un conjunto de datos ("nueve niños tienen familias de cuatro personas")" (NCTM, 2003, p. 113). 
- "Deberían discutir cuándo se pueden aplicar o no las conclusiones obtenidas de los datos de una población, a otra población. Las consideraciones de ese tipo son las precursoras de la comprensión de la noción de inferencia muestral" (NCTM, 2003, p. 113).

- "Apreciar las semejanzas y diferencias existentes en dos conjuntos de datos, requiere que los alumnos lleguen a ser más precisos al describirlos. Así, se va desarrollando la idea de valor "típico" o promedio. Y, a partir de la comprensión informal de "el que más" y el "mediano", los alumnos pueden llegar a las nociones de moda, mediana, e, informal mente, de media" (NCTM, 2003, p. 183-184).

- "Es también necesario que los alumnos piensen sobre las medidas de centralización en relación con la dispersión de una distribución" (NCTM, 2003, p. 255).

Argumentos:

En relación a los argumentos se detallan a continuación las normas y explicaciones de las mismas extraídas del análisis de los PE-2000; en este caso hemos encontrado unidades de análisis que hacen referencia a las matemáticas en general, no encontrando enunciados que hiciesen referencia de manera específica a las argumentaciones en estadística.

Normas:

- "Elegir y utilizar varios tipos de razonamiento y métodos de demostración" (NCTM, 2003, p. 192).

- "Los alumnos deberían formular con frecuencia conjeturas sobre relaciones matemáticas, investigar estas conjeturas y elaborar argumentos matemáticos con base en su trabajo" (NCTM, 2003, p. 195).

Estas normas y otras similares pueden ser refundidas en las siguientes normas de carácter general (estándar de proceso dedicado al razonamiento y la demostración):

Los programas de enseñanza de todas las etapas deberían capacitar a todos los estudiantes para:

- reconocer el razonamiento y la prueba como aspectos fundamentales de las matemáticas;

- hacer e investigar conjeturas matemáticas;

- desarrollar y evaluar argumentos y pruebas;

- seleccionar y usar varios tipos de razonamientos y métodos de prueba. 


\section{Explicación:}

- "Parte del razonamiento matemático consiste en examinar y tratar de comprender por qué algo que parece ser cierto, no lo es, y en empezar a usar contraejemplos en este contexto" (NCTM, 2003, p. 195).

- "utilizar los razonamientos inductivo y deductivo para formular argumentos matemáticos" (NCTM, 2003, p. 266).

\section{Relaciones entre componentes epistémicos}

En el análisis de los PE-2000 hemos encontrado también enunciados que hacen referencia a relaciones entre varios componentes de la idoneidad epistémica, por lo que no podían ubicarse únicamente dentro de las unidades de análisis pertenecientes a uno de dichos componentes. Indicamos a continuación algunas de dichas unidades de análisis en las cuales se mencionan relaciones que se deben establecer entre los distintos componentes de la dimensión epistémica:

- "destacar también la importancia de los conceptos matemáticos para la resolución de problemas" (NCTM, 2003, p. 16). (Conecta elementos regulativos con problemas)

- "La resolución de problemas puede y debería utilizarse para ayudar a los estudiantes a desarrollar fluidez con destrezas específicas" (NCTM, 2003, p. 55). (Vincula problemas con conceptos, procedimientos y argumentos)

- "Las representaciones deberían tratarse como elementos esenciales para sustentar la comprensión de los conceptos y relaciones matemáticos" (NCTM, 2003, p. 71). (Vincula lenguaje con conceptos y procedimientos)

- "En la etapa 3-5, deberían crear representaciones más detalladas y seguras. Su repertorio de símbolos, herramientas y notaciones convencionales debería ampliarse, y estar claramente conectado a los conceptos que exploran" (NCTM, 2003, p. 211). (Vincula lenguaje con conceptos)

El estándar de proceso sobre conexiones matemáticas sintetiza claramente la posición de los PE-2000 sobre la necesidad de establecer conexiones entre las ideas matemáticas, tanto internas a la propia matemática como con otros contextos. Las anteriores conexiones encontradas se unifican en las siguientes normas que hacen referencia a conexiones entre las distintas componentes de la idoneidad epistémica:

Los programas de enseñanza de todas las etapas deberían capacitar a todos los estudiantes para: 
- reconocer y usar conexiones entre las ideas matemáticas;

- comprender cómo se relacionan las ideas matemáticas y se organizan en un todo coherente;

- reconocer y aplicar las ideas matemáticas en contextos no matemáticos.

\section{Síntesis de indicadores de idoneidad epistémica}

De acuerdo con la Teoría de la Idoneidad Didáctica, un proceso de instrucción tiene mayor idoneidad epistémica en la medida en que los significados pretendidos (o implementados) constituyen una muestra representativa de un significado de referencia. Esta noción de significado de referencia se interpreta en términos de las prácticas operativas y discursivas puestas en juego en la resolución de cierto tipo de situaciones-problemas, que se consideran abordables y adecuadas para un cierto nivel educativo por parte de la comunidad educativa en que tiene lugar el proceso instruccional. El sistema de prácticas operativas y discursivas incluye la configuración de objetos y procesos puestos en juego en su realización, y por tanto la trama de funciones semióticas (conocimientos) que los relacionan (GODINO; BATANERO; FONT, 2007).

Las normas epistémicas que hemos identificado en los PE-2000 se pueden interpretar como la concreción del significado de referencia que la comunidad educativa (investigadores, expertos y formadores de profesores de matemáticas) que ha producido los PE-2000 propone para los procesos de instrucción matemática. Aquellos procesos que son diseñados e implementados de acuerdo a esta referencia se consideran como de máxima idoneidad desde el punto de vista del contenido matemático.

En el cuadro 2 presentamos una síntesis de los indicadores de idoneidad epistémica inferidos a partir del análisis de los PE-2000 en relación a las matemáticas en general, que se obtienen interpretando las normas epistémicas de la sección 3 en términos de indicadores y refundiendo las normas, explicaciones y justificaciones que consideramos similares o que aportan ligeras variantes. A dicha síntesis de indicadores la denominamos Pauta-NCTM. 
Cuadro 2 - Indicadores de idoneidad epistémica en los PE-2000 (cualquier contenido matemático)

Problemas:

a') Se utiliza la resolución de problemas como un medio para introducir, ampliar y aplicar el conocimiento.

b’) Se plantean problemas, que surjan de las matemáticas y de otros contextos, que implican establecer relaciones y que favorecen el desarrollo de diferentes estrategias.

Lenguajes/representaciones:

a') Se usa un amplio repertorio de representaciones (materiales, icónicas y simbólicas) para modelizar problemas e ideas matemáticas, analizando la pertinencia y potencialidad de uno u otro tipo de representación y realizando procesos de traducción entre las mismas.

b')Se favorece que los estudiantes construyan, perfeccionen y usen sus propias representaciones para organizar, registrar y comunicar ideas.

Reglas (conceptos, procedimientos, proposiciones):

a) Se introducen y aplican conceptos y procedimientos con profundidad y comprensión.

b’) Se aplican y adaptan diversas estrategias para resolver problemas.

Argumentos:

a') Se favorece el razonamiento y la prueba de los enunciados y proposiciones matemáticas mediante diversos tipos de razonamientos y métodos de prueba.

b’) Los estudiantes formulan con frecuencia conjeturas sobre relaciones matemáticas, las investigan y justifican.

Relaciones:

a') Se favorece el establecimiento y el uso de conexiones entre las ideas matemática (problemas, representaciones, conceptos, procedimientos, propiedades argumentos)

b’) Los contenidos matemáticos se presentan y estudian como un todo organizado.

c') Se reconocen y aplican las ideas matemáticas en contextos no matemáticos.

Fuente: Elaboración propia.

\section{Comparación de los indicadores de las pautas EOS y NCTM: una versión revisada}

Una vez obtenida la Pauta-NCTM, instrumento basado en la información contenida en los PE-2000, se hace necesaria la comparación de dicha pauta con la pauta más general, la Pauta-EOS (GODINO, 2011). Se pretende comparar los indicadores presentes en ambos instrumentos con el fin de complementar ambas pautas y obtener un conjunto de indicadores revisados. A continuación se realiza dicha comparación mostrando los comentarios y resultados dentro de cada uno de los distintos componentes de la idoneidad epistémica.

Situaciones-problemas

La comparación entre los indicadores de las pautas EOS y NCTM en el componente situacional (resolución de problemas) nos lleva a proponer el siguiente enunciado como una versión refinada: 
a") Se presenta una muestra representativa y articulada de situaciones-problemas que permitan contextualizar, ejercitar, ampliar y aplicar el conocimiento matemático, los cuales proceden de la propia matemática y de otros contextos.

El indicador b) aporta un matiz propio que resalta el origen de los problemas, los cuales también deberían ser propuestos por los propios estudiantes.

Lenguajes / representaciones

Las formulaciones a') y b') refieren a un contenido similar, pero son más explícitas y detalladas que las correspondientes a) y b). El indicador b) incorpora un aspecto nuevo y relevante: la necesidad de que el lenguaje sea adecuado a los estudiantes a los que se dirige. Los indicadores revisados para el componente representacional quedarían:

a”) Se usa un amplio repertorio de representaciones (materiales, icónicas y simbólicas) para modelizar problemas e ideas matemáticas, analizando la pertinencia y potencialidad de uno u otro tipo de representación y realizando procesos de traducción entre las mismas.

b") Se favorece que los estudiantes construyan, perfeccionen y usen sus propias representaciones para organizar, registrar y comunicar ideas.

c”) El nivel del lenguaje usado es adecuado a los estudiantes a que se dirige.

\section{Elementos regulativos}

En cuanto a los elementos regulativos los indicadores a), b) y c) incluyen aspectos que no quedan reflejados en los a') y b'); resaltan que se incluyan en el programa de instrucción las definiciones y procedimientos fundamentales y que sean formulados de manera correcta, clara y adaptados al nivel educativo (lo cual facilitará la comprensión). El indicador c) responde al interés de tener en cuenta las ideas previas de los estudiantes.

Argumentos

Los indicadores a') y b') desarrollan de manera más explícita y precisa el indicar b), mientras que el a) incorpora un aspecto que no queda reflejado en los a') y b'): dado que los enunciados se pueden justificar de maneras diversas, será necesario que se seleccionen aquellas justificaciones más adaptadas al nivel educativo en que tiene lugar el proceso de instrucción. La pauta revisada quedaría para este componente con tres indicadores. 
Relaciones entre componentes epistémicos:

Los indicadores a'), b’) y c') desarrollan de manera más explícita y precisa el enunciado a) sobre relaciones incluido en la Pauta-EOS.

En lo cuadro 3 incluimos los indicadores de idoneidad epistémica resultante de la comparación de la Pauta-EOS y Pauta-NCTM.

Cuadro 3 - Indicadores revisados de idoneidad epistémica

\begin{tabular}{|c|c|}
\hline COMPONENTES & INDICADORES \\
\hline Situaciones-problemas & $\begin{array}{l}\text { a") Se presenta una muestra representativa y articulada de } \\
\text { situaciones-problemas que permitan contextualizar, ejercitar, } \\
\text { ampliar y aplicar el conocimiento matemático, los cuales } \\
\text { proceden de la propia matemática y de otros contextos. } \\
\text { b") Se proponen situaciones de generación de problemas } \\
\text { (problematización). }\end{array}$ \\
\hline Lenguajes & $\begin{array}{l}\text { a") Se usa un amplio repertorio de representaciones (materiales, } \\
\text { icónicas y simbólicas) para modelizar problemas e ideas } \\
\text { matemáticas, analizando la pertinencia y potencialidad de uno u } \\
\text { otro tipo de representación y realizando procesos de traducción } \\
\text { entre las mismas. } \\
\text { b”) Se favorece que los estudiantes construyan, perfeccionen } \\
\text { y usen sus propias representaciones para organizar, registrar y } \\
\text { comunicar ideas. } \\
\text { c") El nivel del lenguaje usado es adecuado a los estudiantes a } \\
\text { que se dirige. }\end{array}$ \\
\hline $\begin{array}{l}\text { Reglas (Definiciones, } \\
\text { proposiciones, } \\
\text { procedimientos) }\end{array}$ & $\begin{array}{l}\text { a") Las definiciones y procedimientos son claros y correctos, y } \\
\text { están adaptados al nivel educativo al que se dirigen. } \\
\text { b") Se presentan los enunciados y procedimientos fundamentales } \\
\text { del tema adaptados al nivel educativo dado. } \\
\text { c") Se proponen situaciones donde los alumnos tengan que } \\
\text { generar o negociar definiciones, proposiciones o procedimientos. }\end{array}$ \\
\hline Argumentos & $\begin{array}{l}\text { a") Se favorece el razonamiento y la prueba de los enunciados } \\
\text { y proposiciones matemáticas mediante diversos tipos de } \\
\text { razonamientos y métodos de prueba. } \\
\text { b") Los estudiantes formulan con frecuencia conjeturas sobre } \\
\text { relaciones matemáticas, las investigan y justifican. } \\
\text { c") Las explicaciones, comprobaciones y demostraciones son } \\
\text { adecuadas al nivel educativo a que se dirigen. }\end{array}$ \\
\hline Relaciones & $\begin{array}{l}\text { a") Se favorece el establecimiento y el uso de conexiones entre } \\
\text { las ideas matemática (problemas, representaciones, conceptos, } \\
\text { procedimientos, propiedades argumentos). } \\
\text { b") Los contenidos matemáticos se presentan y estudian como } \\
\text { un todo organizado. } \\
\text { c") Se reconocen y aplican las ideas matemáticas en contextos } \\
\text { no matemáticos. }\end{array}$ \\
\hline
\end{tabular}

Fuente: Elaboración propia. 


\section{Indicadores de idoneidad epistémica específicos del estudio del análisis de datos y probabilidad}

En el cuadro 4 sintetizamos el conjunto de indicadores de idoneidad epistémica inferidos de los PE-2000 para el bloque de contenido de análisis de datos y probabilidad. Dicho conjunto de indicadores es una aportación útil para el diseño y evaluación de planes de formación en estadística en los niveles de K-8. Queda pendiente para próximos trabajos la comparación de este sistema con otras propuestas curriculares y resultados de la investigación sobre educación estadística.

Cuadro 4 - Indicadores de idoneidad epistémica en los PE-2000 (contenido de estadística)

Problemas:

a) Se plantean problemas - investigaciones (proyectos) con diversas fuentes y tipos de datos teniendo en cuenta los elementos básicos del análisis estadístico elemental: formulación de preguntas, selección y descripción de la muestra, establecimiento de hipótesis, recogida de datos (método de encuesta, experimento u observación), organización de los datos y formulación de inferencias.

Lenguajes:

a) Se utilizan diferentes representaciones de uso convencional en estadística y probabilidad, tales como: números, símbolos, coordenadas cartesianas, palabras, frecuencias absolutas, frecuencias relativas, tablas, histogramas y diagramas (de barra, lineal, de sectores, de caja y de puntos). Los gráficos incluyen los títulos y etiquetas que permiten identificar claramente los datos representados.

b) Se incluyen situaciones en las que se requiere discriminar entre el uso más adecuado de una u otra representación estadística.

Reglas (conceptos, procedimientos, proposiciones):

a) Se analizan, describen y comparan conjuntos de datos calculando estadísticos (media, mediana, moda, desviación típica, rango, rango intercuartílico y valor atípico), construyendo gráficos (histogramas y gráficos de barra, de línea, de sectores, de tronco, de caja y de nubes de puntos con líneas de ajuste) y estableciendo la relación entre las medidas de centralización en relación con la dispersión de una distribución.

b) Se describen y evalúan las posibilidades de ocurrencia de un suceso como posible, imposible, probable o seguro, a partir de experiencias cercanas y de la observación de regularidades en experimentos aleatorios simples.

c) Se introducen la regla de Laplace como un modelo que permite predecir el valor de la probabilidad de ocurrencia de un evento simple, sin realizar el experimento aleatorio.

Fuente: Elaboración propia.

\section{Interacciones entre la faceta epistémica y las restantes facetas}

En los apartados anteriores hemos identificado algunos indicadores de idoneidad para la faceta epistémica. Esta faceta no se debe considerar como 
un factor independiente, ya que de hecho se producen interacciones con las restantes. Así, por ejemplo, el uso de un recurso tecnológico puede determinar que se puedan abordar determinados tipos de problemas y las configuraciones de objetos y procesos correspondientes, lo cual conlleva nuevas formas de representación, argumentación, generalización, etc.

En los PE-2000 hemos encontrado algunos indicadores de interacción entre la faceta epistémica y las restantes, que complementan los propuestos en Godino (2011), especialmente algunos que incluyen especificaciones de interés para el caso de la estadística.Así para la interacción epistémica-ecológica resaltamos:

- Se conectan los contenidos de estadística y probabilidad con los de otras áreas de la matemática escolar.

En la interacción entre las facetas epistémica y cognitiva encontramos el siguiente indicador:

- Se utiliza la resolución de problemas para favorecer procesos meta cognitivos.

En la interacción epistémica-afectiva encontramos conveniente incorporar el indicador:

- Se plantean problemas relacionados con los intereses de los estudiantes.

- Los profesores manifiestan confianza en las capacidades de sus estudiantes y valoran su trabajo como una forma de incentivarles a resolver problemas y comunicar sus resultados.

En la interacción epistémica-interaccional encontramos los siguientes indicadores que involucra matices relevantes respecto de los considerados en la faceta epistémica:

- En las discusiones de clase, se analizan las diferentes representaciones utilizadas para organizar y exponer datos y se compara su eficacia.

- El profesor brinda oportunidades a los estudiantes para que formulen y desarrollen sus propios problemas e investigaciones de manera individual y grupal.

- Los profesores fomentan maneras de justificar que estén al alcance de los alumnos.

- Los profesores generan instancias donde los estudiantes puedan comunicar sus ideas matemáticas en forma escrita y oral.

Respecto de la faceta mediacional y su relación con la faceta epistémica encontramos como indicador complementario:

- Se analizan las características específicas de las representaciones semióticas que suministran las tecnologías. 


\section{Indicadores de idoneidad ecológica}

La idoneidad ecológica se refiere al grado en que un plan o acción formativa para aprender matemáticas resulta adecuado dentro del entorno en que se utiliza. Por entorno se entiende todo lo que está fuera del aula, condicionando la actividad que se desarrolla en la misma. Así, nos podemos referir a todo lo que viene en general condicionado por la sociedad, la escuela, la pedagogía, la didáctica de las matemáticas. El proceso de estudio tiene lugar en un contexto educativo que fija unos fines y valores para la educación de los ciudadanos y profesionales que se deben respetar. Dichos fines y valores son interpretados y especificados dentro del proyecto educativo del centro o departamento que coordina la acción de los distintos profesores implicados.

En el análisis que hemos realizado de los PE-2000 hemos encontrado algunos indicadores para la faceta ecológica que complementan a los propuestos en Godino (2011). En el componente, "Adaptación al currículo" consideramos de interés destacar el indicador:

- Se actúa con flexibilidad adaptando convenientemente algunos elementos del currículo.

Este indicador se relaciona con la adaptación a la diversidad de intereses y capacidad de los alumnos, aspecto que resaltan las diversas orientaciones curriculares.

Para el caso de la estadística hemos inferido de los PE-2000 indicadores de interés para las adaptaciones socio-profesionales, así como para las conexiones intra e interdisciplinares:

- Se estudian aplicaciones de la estadística y la probabilidad en diferentes contextos de la vida cotidiana.

- Se trabajan diferentes ideas de la estadística y la probabilidad conectadas entre sí, relacionando las áreas de un mismo nivel y las conexiones que se dan entre niveles.

\section{Reflexiones finales e implicaciones}

Una de las aportaciones de este trabajo es entender que aunque sea posible elaborar "una pauta" genérica que sintetice los principales criterios de idoneidad provenientes de la investigación didáctica (tal es el propósito de la Pauta-EOS), en realidad pueden elaborarse pautas más locales derivadas de propuestas curriculares, modelos teóricos determinados, o también que desarrollen criterios referidos a contenidos o niveles educativos específicos. Este es el caso de la Pauta-NCTM, que hemos elaborado a partir del análisis del contenido de los PE-2000. 
Mediante la metodología aplicada en este trabajo hemos "filtrado" las orientaciones curriculares de los PE-2000 a través de las categorías epistémicas, cognitivas e instruccionales que propone el EOS, "destilando" de esta manera el sistema de indicadores de idoneidad que caracterizan dichas orientaciones. Este análisis, aplicado a otras directrices curriculares producirá diferentes pautas, por lo que se plantea la tarea de su comparación y posible articulación.

Las pautas pueden ser vistas como síntesis de conocimientos didáctico-matemáticos (GODINO, 2009) a tener en cuenta en el diseño, implementación y evaluación de procesos de instrucción matemática. Son listas de control que apoyan la reflexión sistemática sobre las diversas facetas y componentes que intervienen o definen dichos procesos. Constituyen, por tanto, un puente entre el complejo de información didáctica disponible (contenida en múltiples informes de investigación, documentos curriculares, recursos instruccionales,...) y la práctica de la enseñanza.

En consecuencia estas pautas se pueden usar como "artefactos" o recursos en la formación de profesores; si los futuros profesores se apropiaran de estos artefactos (lo que implica conocerlas, comprenderlas y lograr competencia en su aplicación, esto es, convertirlos en instrumentos de trabajo personal), podrían utilizarlas en el diseño, implementación y evaluación de sus propias experiencias docentes $^{7}$. Las pautas dan criterios o normas sobre las características de un proceso de instrucción matemática a implementar (o evaluar) con estudiantes de matemáticas. Al mismo tiempo tales criterios pueden ser vistos como síntesis de conocimientos didáctico-matemáticos que deben conocer (comprender y usar de manera competente) los profesores para que puedan lograr alta idoneidad en los procesos instruccionales que ellos diseñen e implemente (figura 1).

Figura 1 - Pautas de idoneidad como artefactos mediadores

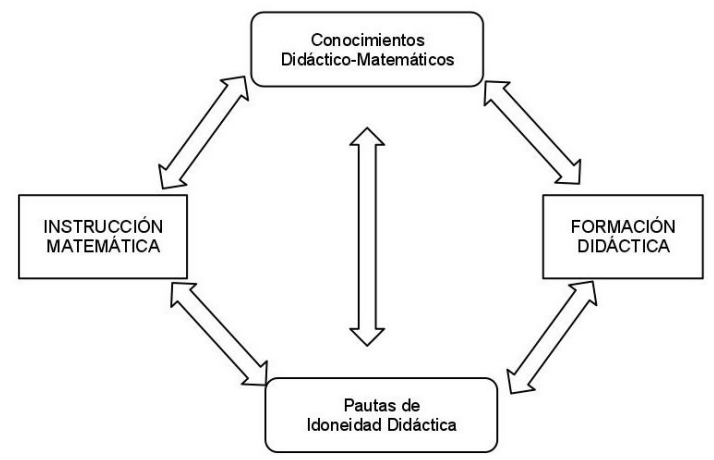

Fuente: Elaboración propia.

\footnotetext{
${ }^{7}$ En Arteaga (2010) se describe una experiencia de uso de los indicadores de idoneidad didáctica por futuros profesores de educación primaria como medio de apoyar la reflexión sistemática sobre una experiencia formativa.
} 
Es importante resaltar que la aplicación concreta de las reglas o criterios de idoneidad es «situada». Es decir, la aplicación, priorización, relegación, etc., de dichas reglas depende del contexto institucional en el que se desarrolla el proceso de instrucción y del criterio pedagógico y didáctico del profesor que las debe tener en cuenta. Se trata de guías de orientación para la mejora de los procesos de instrucción, no de un criterio que produzca la frustración del profesor «normal» al no poderlo alcanzar (GODINO et al., 2009, p. 61).

Reconocimientos:

Trabajo realizado en el marco del proyecto de investigación, EDU2010-14947, Ministerio de Ciencia e Innovación (Madrid).

\section{Referencias}

ARTEAGA, P. Evaluación de conocimientos sobre gráficos estadísticos y conocimientos didácticos de futuros profesores. 2011. 433 f. Tesis doctoral (Didáctica de la Matemática) - Universidad de Granada, Granada, 2011. Disponible en: < http://www. ugr.es/local/batanero>. Acceso en: 28 mayo 2012.

BROUSSEAU, B. Theory of didactical situations in mathematics. Dordrecht: Kluwer, 1997.

FREUDENTHAL, H. Revisiting mathematics education: China lectures. Dordrecht: Kluwer, 1991.

GODINO, J. D. Un enfoque ontológico y semiótico de la cognición matemática. Recherches en Didactiques des Mathematiques, v. 22, n. 2-3, p. 237-284, 2002.

. Categorías de análisis de los conocimientos del profesor de matemáticas. UNIÓN -

Revista Iberoamericana de Educación Matemática, n. 20, p. 13-31, 2009.

Indicadores de la idoneidad didáctica de procesos de enseñanza y aprendizaje de las matemáticas. In: CONFERÊNCIA INTERAMERICANA DE EDUCAÇÃO MATEMÁTICA - CIAEM, 13., 2011, Recife. Anais... Recife, 2011. Disponible en: <http:// www.ugr.es/local/jgodino>. Acceso en: 28 mayo 2012.

GODINO, J. D.; BATANERO, C.; FONT, V. The onto-semiotic approach to research in mathematics education. The International Journal on Mathematics Education, ZDM, v. 39, n. 1-2, p. 127-135, 2007.

GODINO, J. D.; BENCOMO, D.; FONT, V.; WILHELMI, M. R. Análisis y valoración de la idoneidad didáctica de procesos de estudio de las matemáticas. Paradigma, v. 27, n. 2, p. 221-252, dic. 2007.

GODINO,J.D.; FONT, V.; WILHELMI, M. R.;DE CASTRO, C. Aproximación a la dimensión normativa en Didáctica de la Matemática desde un enfoque ontosemiótico. Enseñanza de las Ciencias, v. 27, n. 1, p. 59-76, 2009. 
HJALMARSON, M. A.; LESH, R. Design research. Engineering, systems, products, and processes for innovation. In: ENGLISH, L. D. (Ed.). Handbook of international research in mathematics education. New York: Routledge, 2008. p. 520-534.

LESH, R.; SRIRAMAN, B. Re-conceptualizing mathematics education as a design science. In: SRIRAMAN, B.; ENGLISH, L. (Eds.). Theories of Mathematics Education: seeing new frontiers. Heidelberg: Springer, 2010. p. 123-146.

MEC. Real Decreto $\mathrm{n}^{\mathbf{0}} \mathbf{1 5 1 3 / 2 0 0 6}$, de 7 de diciembre, por el que se establecen las enseñanzas mínimas correspondientes a la Educación Primaria. Madrid: MEC, 2006.

NATIONAL COUNCIL OF TEACHERS OF MATHEMATICS. Principios y estándares para la Educación Matemática. Traducción de M. Fernández (Traducción de la versión del 2000 del NCTM). Reston, VA: NCTM, Sociedad Andaluza de Educación Matemática Thales, 2003.

REIGELUTH, C. M. ¿En qué consiste una teoría de diseño educativo y cómo se está transformando? In: REIGELUTH, C. M. (Ed.). Diseño de la instrucción teorías y modelos: un nuevo paradigma de la teoría de la instrucción. Madrid: Santillana, 2000. p. $15-40$.

RIVAS, H. Valoración de la idoneidad didáctica de procesos de formación estadística para profesores de educación primaria. 2011. Tesis de Fin de Máster (Didáctica de la Matemática) - Universidad de Granada, Granada, 2011.

WITTMAN, E. C. Mathematics education as a 'design science'. Educational Studies in Mathematics, v. 29, n. 4, p. 355-374, 1995.

Recebido em 01/03/2012

Aceito em 15/05/2012 